\title{
Mapping quantitative trait loci with additive effects and additive $x$ additive epistatic interactions for biomass yield, grain yield, and straw yield using a doubled haploid population of wheat (Triticum aestivum L.)
}

\author{
Z.K. Li ${ }^{1}$, X.L. Jiang ${ }^{2}$, T. Peng ${ }^{3}$, C.L. Shi ${ }^{1}$, S.X. Han ${ }^{1}$, B. Tian ${ }^{1}$, Z.L. Zhu ${ }^{1}$ \\ and J.C. Tian ${ }^{1}$ \\ ${ }^{1}$ State Key Laboratory of Crop Biology, Shandong Agricultural University, \\ Tai'an, China \\ ${ }^{2}$ Center of Wheat Breeding, Henan Institute of Science and Technology, \\ Xinxiang, China \\ ${ }^{3}$ Institute of Jiyuan Agricultural Science, Jiyuan, China \\ Corresponding author: J.C. Tian \\ E-mail: xiao_ling_jiang@163.com \\ Genet. Mol. Res. 13 (1): 1412-1424 (2014) \\ Received January 21, 2013 \\ Accepted August 1, 2013 \\ Published February 28, 2014 \\ DOI http://dx.doi.org/10.4238/2014.February.28.14
}

\begin{abstract}
Biomass yield is one of the most important traits for wheat (Triticum aestivum L.)-breeding programs. Increasing the yield of the aerial parts of wheat varieties will be an integral component of future wheat improvement; however, little is known regarding the genetic control of aerial part yield. A doubled haploid population, comprising 168 lines derived from a cross between two winter wheat cultivars, 'Huapei 3' (HP3) and 'Yumai 57' (YM57), was investigated. Quantitative trait loci (QTL) for total biomass yield, grain yield, and straw yield were determined for additive effects and additive $\mathrm{x}$ additive epistatic interactions using the QTLNetwork 2.0 software based on the
\end{abstract}


mixed-linear model. Thirteen QTL were determined to have significant additive effects for the three yield traits, of which six also exhibited epistatic effects. Eleven significant additive $\mathrm{x}$ additive interactions were detected, of which seven occurred between QTL showing epistatic effects only, two occurred between QTL showing epistatic effects and additive effects, and two occurred between QTL with additive effects. These QTL explained 1.20 to $10.87 \%$ of the total phenotypic variation. The QTL with an allele originating from YM57 on chromosome 4B and another QTL contributed by HP3 alleles on chromosome 4D were simultaneously detected on the same or adjacent chromosome intervals for the three traits in two environments. Most of the repeatedly detected QTL across environments were not significant $(\mathrm{P}>0.05)$. These results have implications for selection strategies in wheat biomass yield and for increasing the yield of the aerial part of wheat.

Key words: Wheat; Biomass yield; Grain yield; Straw yield; Additive; Epistatic

\section{INTRODUCTION}

By 2020, wheat production will have to increase by $40 \%$ in order to meet requirements with respect to human food and animal feed, which will mainly be accomplished by increasing yield (Pfeiffer et al., 2000). "Increasing the intensity of production in those ecosystems that lend themselves to sustainable intensification, while decreasing intensity of production in the more fragile ecosystems" may be the only way for agriculture to keep pace with increasing population growth (Borlaug and Dowswell, 1997). Hence, future wheat improvement strategies must emphasize biomass yield (BY), grain yield (GY), and straw yield (SY) in concert. $\mathrm{BY}$ is an important trait for wheat improvement and for its contribution to the world's economy. GY is a particularly complex trait, being the end product of many processes in the plant and, in consequence, is very environmentally dependent (Quarrie et al., 2005). GY forms one of the key economic drivers behind successful wheat production, and is consequently a major target for wheat-breeding programs (Kuchel et al., 2007). SY, an important by-product in the production of agricultural crops, is considered as a potentially considerable source of renewable energy supply with an estimated value of $47 \times 10^{18} \mathrm{~J}$ worldwide (Lal, 2005). Recently, the straw of crops has received renewed attention, resulting in substituting straw for natural gas or marsh gas instead of simply burning it. The main benefits of this practice lie in the conservation of non-renewable resources and in the reduction of greenhouse gas emissions (Gabrielle and Gagnaire, 2008). Therefore, understanding the genetic architecture for the aerial part of BY, GY, and SY will be of importance to wheat improvement.

The application of molecular marker technologies for quantitative trait loci (QTL) analysis has provided an effective approach to dissect complicated quantitative traits and to study their relative effects (Doerge, 2002). Genetic dissection of yield components can help to elucidate the physiological route from gene to phenotype for BY, GY, and SY (Kuchel et al., 2007). Several genetic associations with GY have been reported to date in various crops, including maize (Li et al., 2010), rice (Huang et al., 2009), barley (Xue et al., 2010), and wheat 
(Kuchel et al., 2007; Maccaferri et al., 2008). However, yield component studies reporting QTL that are associated with biomass and straw are relatively rare, although they have both been shown to be important for GY in limited environments (McIntyre et al., 2010). Li et al. (2001) reported QTL influencing rice biomass yield in RILs, BC, and testcross mapping populations, demonstrating a few main-effect QTL and a large number of epistatic QTL pairs associated with biomass and straw traits.

Ayala et al. (2002) discovered five QTL for biomass on chromosomes 2B, 2D, 3B, 4D, and 6A under artificial inoculation with a BYDV-PAV-Mex isolate and under disease-free conditions in two wheat populations, Opata x Synthetic (ITMI) and Frontana x INIA66 (F x I). Zhang et al. (2004) detected four QTL ( $q B M-1-1, q B M-1-2, q B M-3$, and $q B M-5)$ with $R^{2}$ values ranging from 13 to $28 \%$ for biomass in a rice doubled haploid (DH) population. Liu et al. (2006) showed additive and additive by additive epistatic QTL with significant effects on rice biomass yield and its two component traits (SY and GY), using a population of 125 DH lines from an inter-subspecific cross of IR64 and Azucena. Four QTL and one pair of epistatic QTL were also found to be responsible for the positive correlation between BY and SY. Kirigwi et al. (2007) mapped some QTL for biomass production and biomass production rate on the proximal region of chromosome $4 \mathrm{AL}$ in wheat under differing soil moisture regime treatments. Anhalt et al. (2009) mapped QTL contributing to BY traits, including dry weight and dry matter in an $\mathrm{F}_{2}$ population consisting of 360 individual genotypes. A major QTL with additive effects was found on LG3 to be largely responsible for BY traits in Lolium perenne L. (perennial ryegrass). Consequently, present knowledge of the genetic relationships among BY traits remains fragmentary.

With the objective of better understanding the genetic basis for yield potential associated with BY, GY, and SY, additive and additive $\mathrm{x}$ additive epistatic QTL were investigated based on the mixed linear model in the present study, using a DH population comprising 168 lines, derived from a cross between two winter wheat cultivars, 'Huapei 3' (HP3) and 'Yumai 57' (YM57). The relationships of the total biomass, grain, and straw yields were analyzed at QTL levels. The results will be of great significance for helping breeders to enhance the yield of wheat.

\section{MATERIAL AND METHODS}

\section{Plant materials}

A population of $168 \mathrm{DH}$ lines was established through microspore culture from a cross between HP3 and YM57 (Guo et al., 2004; Hai and Kang, 2007). HP3 is an elite variety with early maturity, high yield, and high resistance to several diseases (Hai and Kang, 2007), whereas YM57 is widely cultivated for its long growth duration and yield stability under various ecological conditions (Guo et al., 2004). Both wheat cultivars, also differing in important basic quality parameters (Guo et al., 2004; Hai and Kang, 2007), have been planted over large areas in the Yellow and Huai River Valleys' Winter Wheat Zone of China.

\section{Field experiments and linkage map}

Field experiments were conducted in Tai' an, Shandong Province $\left(116^{\circ} 36^{\prime} \mathrm{E}, 36^{\circ} 57^{\prime} \mathrm{N}\right)$ and Jiyuan, Henan Province $\left(112^{\circ} 36^{\prime} \mathrm{E}, 35^{\circ} 05^{\prime} \mathrm{N}\right)$ in 2008 . Shandong and Henan Provinces are 
the main growing areas for winter wheat in China. Field planting followed a randomized complete block design with the DH population and the parents in each trial. Each plot consisted of three rows. There were 20 plants in each row, with a distance of $10 \mathrm{~cm}$ between plants within each row and $25 \mathrm{~cm}$ between rows. The soil was brown earth that contained 40.2, 51.3, and $70.8 \mathrm{mg} / \mathrm{kg}$ available N, P, and K, respectively. The field management followed local standard practices. Ten plants in the middle of the inner two rows of each plot were individually harvested from the soil surface to measure BY per plant in terms of the total dry weight $(\mathrm{g})$ of the entire plant, GY in terms of the total dry weight (g) of grains from the entire plant, and SY in terms of the total dry weight $(\mathrm{g})$ of straws from the entire plant. Trait measurements were averaged over ten plants within each plot for determining differences in statistical analyses.

A genetic linkage map of the DH population that was built in 2008 contains 323 molecular markers, including 284 simple sequence repeat (SSR) loci, 37 expressed sequence tag-SSR loci, one inter-simple sequence repeat locus, and one high molecular weight locus. Twelve markers remain unlinked. The map covered a length of $2485.7 \mathrm{cM}$ with an average distance of $7.67 \mathrm{cM}$ between adjacent markers (Zhang et al., 2009). These markers formed 24 linkages. The chromosomal locations and orders of the markers in the map were in accordance with those reported for Triticum aestivum L. (Somers et al., 2004).

\section{Statistical analysis}

Phenotypic and correlation analyses were conducted using SPSS version 13.0 (SPSS, Chicago, IL, USA). QTL analysis was performed using the QTLNetwork2.0 software (Yang and Zhu, 2005) based on the mixed linear model (Wang et al., 1999). Composite interval analysis was undertaken using forward-backward stepwise multiple linear regression with a probability in and out of the model ranging from 0.05 to 0.5 , and a window size set at $10 \mathrm{cM}$. Significant thresholds for QTL detection were calculated for each dataset using 1000 permutations and a genome-wide error rate of 0.05 (significant). The final genetic model incorporated significant additive and epistatic effects as well as their environmental interactions. A significant QTL was identified if the phenotype was associated with a marker locus at $\mathrm{P}<0.005$.

\section{RESULTS}

\section{Phenotypic performance of DH lines and parents}

The phenotypic variation among DH lines and the parents of BY, GY, and SY measured under two environments in 2008 are summarized in Table 1 and Figure 1. HP3 and YM57 differed significantly in the measured traits, with phenotypic values of HP3 for BY, GY, and SY being much higher than those of YM57. Some lines had more extreme values than the parents in both environments, showing substantial transgressive segregation, although the average values of $\mathrm{DH}$ lines for those traits were intermediate between the parental values. In addition, the three traits showed considerable phenotypic variation and continuous distributions, indicating their quantitative nature. Both the skew and kurt of BY, GY, and SY were less than 1.0, implying polygenic inheritance and suitability of the data for QTL analysis. Harvest index (grain yield/biomass yield) of DH lines ranged from 0.32 to 0.54 in Tai'an, and ranged from 0.34 to 0.53 in Jiyuan. Both parents had a stable harvest index in the two environments. 
Table 1. Phenotypic summary of biomass yield (BY), grain yield (GY), and straw yield (SY) for HP3 ( $\left.\mathrm{P}_{1}\right)$, YM57 $\left(\mathrm{P}_{2}\right)$, and the doubled haploid (DH) lines at Tai' an and Jiyuan in 2008.

\begin{tabular}{|c|c|c|c|c|c|c|c|c|c|c|}
\hline Environment & Trait & HP3 & YM57 & Means & Min & Max & SD & Skew & Kurt & $\mathrm{HI}$ \\
\hline \multirow[t]{3}{*}{2008 in Tai'an } & BY & 48.52 & 38.68 & 45.82 & 23.53 & 65.34 & 7.17 & -0.14 & -0.18 & \multirow[t]{3}{*}{0.32 to 0.54} \\
\hline & GY & 21.55 & 15.55 & 19.69 & 9.48 & 28.70 & 3.71 & -0.27 & -0.48 & \\
\hline & SY & 26.97 & 23.13 & 26.14 & 14.05 & 41.04 & 4.34 & 0.34 & 0.58 & \\
\hline \multirow[t]{3}{*}{2008 in Henan } & BY & 58.18 & 46.87 & 52.05 & 26.80 & 73.00 & 7.79 & -0.28 & -0.06 & \multirow[t]{3}{*}{0.34 to 0.53} \\
\hline & GY & 26.43 & 19.48 & 22.33 & 9.55 & 30.63 & 3.89 & -0.29 & -0.12 & \\
\hline & SY & 31.75 & 27.39 & 29.72 & 17.25 & 46.75 & 4.78 & 0.45 & 0.90 & \\
\hline
\end{tabular}

$\mathrm{SD}=$ standard deviation; Min $=$ minimum; Max $=$ maximum; Skew $=$ Skewness $;$ Kurt $=$ Kurtosis HI $=$ harvest index.

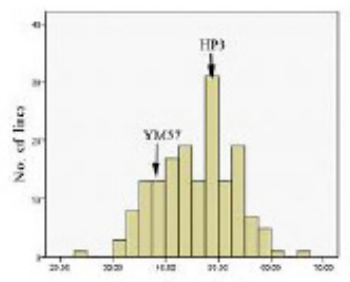

Taikst tiatats gidl
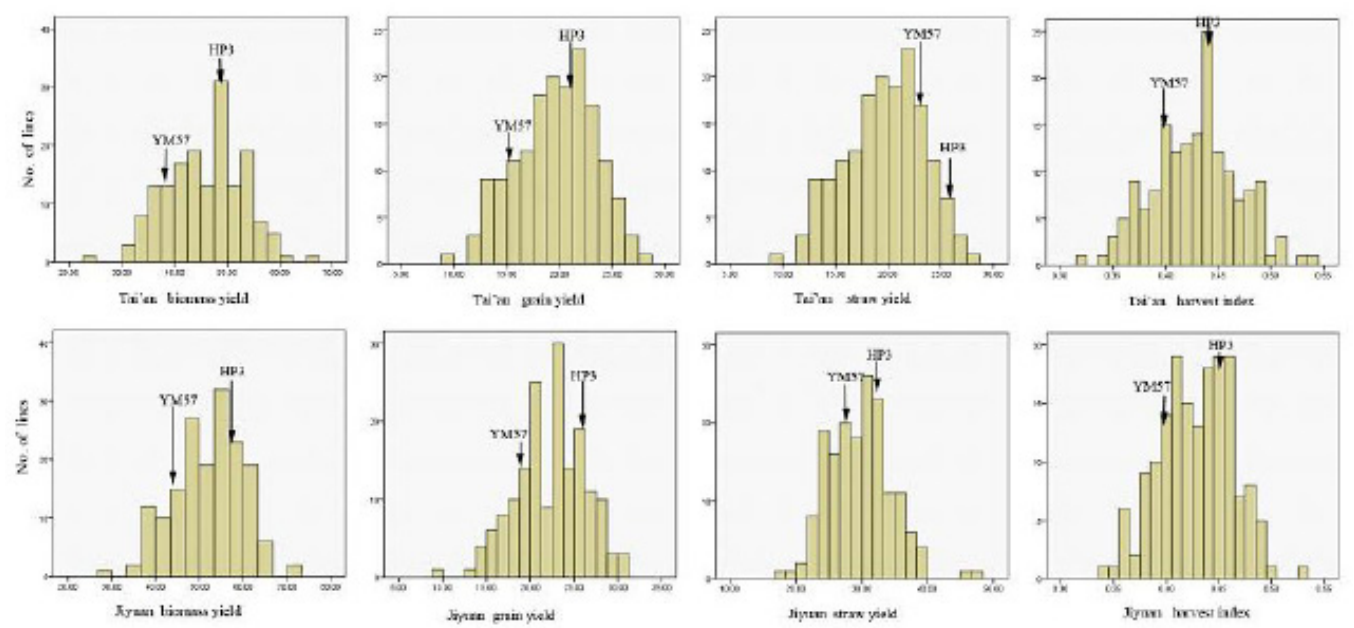

Figure 1. Frequency distributions of BY, GY, and SY for HP3 $\left(\mathrm{P}_{1}\right)$, YM57 $\left(\mathrm{P}_{2}\right)$ and the DH lines at Tai' an and Jiyuan in 2008.

The correlations among BY, GY, and SY are shown in Table 2. The traits were significantly correlated with each other in both environments. BY showed significantly positive correlation with GY $\left(\mathrm{r}^{2}{ }_{1}=0.87^{* *}\right.$ and $\mathrm{r}_{2}{ }_{2}=0.88^{* *}$, where subscripts 1 and 2 represent the environments in Tai' an and Jiyuan, respectively), BY showed significantly positive correlation with SY $(\mathrm{P}<0.01)$, and GY showed significantly positive correlation with $\mathrm{SY}(\mathrm{P}<0.01)$. This showed that both GY and SY played important roles in BY.

Table 2. Coefficients of biomass yield (BY), grain yield (GY), and straw yield (SY) at Tai' an and Jiyuan in 2008.

\begin{tabular}{|c|c|c|c|c|}
\hline \multirow[t]{2}{*}{ Traits } & \multicolumn{2}{|c|}{2008 in Tai'an } & \multicolumn{2}{|c|}{2008 in Henan } \\
\hline & BY & GY & BY & GY \\
\hline $\begin{array}{l}\text { GY } \\
\text { SY }\end{array}$ & $\begin{array}{l}0.87 * * \\
0.91 * *\end{array}$ & $58 * *$ & $0.88^{* *}$ & $0.61 * *$ \\
\hline
\end{tabular}

**Significant at 0.01 probability level. 


\section{QTL mapping} all traits.

Analyses across environments revealed mostly significant genotype interactions for

\section{Additive QTL interactions}

A total of 13 QTL were detected as having significant additive effects for yield traits (Table 3, Figure 2), including four for BY, four for GY, and five for SY. A proportion of these QTL also exhibited epistatic effects for the same trait, which will be described in the next section.

\begin{tabular}{|c|c|c|c|c|c|c|c|}
\hline Traits & Chromosome & QTL & Marker intervals & Position & F value & $\mathrm{A}^{\mathrm{a}}$ & $\mathrm{R}^{2}(\%)^{\mathrm{b}}$ \\
\hline \multirow[t]{4}{*}{ BY } & $3 \mathrm{~A}$ & $Q B y 3 A$ & Xwmc264-Xcfa2193 & 140.9 & 7.66 & 1.45 & 3.5 \\
\hline & $4 \mathrm{~B}$ & $Q B y 4 B$ & Xwmc48-Xbarc1096 & 18.4 & 14.05 & -1.92 & 6.12 \\
\hline & $4 \mathrm{D}$ & $Q B y 4 D$ & Xbarc334-Xwmc331 & 4.1 & 16.06 & 2.56 & 10.87 \\
\hline & $5 \mathrm{~A} 2$ & QBy $5 A 2$ & Xcfe026.1-Xcwem 32.2 & 0 & 7.36 & -1.24 & 2.57 \\
\hline \multirow[t]{4}{*}{ GY } & $2 \mathrm{D}$ & $Q G y 2 D$ & Xgwm539-Xcfd168 & 68.4 & 9.32 & 0.88 & 5.26 \\
\hline & $4 \mathrm{~B}$ & $Q G y 4 B$ & Xwmc48-Xbarc1096 & 18.4 & 12.54 & -0.77 & 4.01 \\
\hline & $4 \mathrm{D}$ & $Q G y 4 D$ & Xbarc334-Xwmc331 & 2.1 & 15.65 & 1.14 & 8.74 \\
\hline & $7 \mathrm{~B} 2$ & $Q G y 7 B 2$ & $X w m c 273.1-X c f d 22.1$ & 10.7 & 8 & -0.8 & 4.29 \\
\hline \multirow[t]{5}{*}{ SY } & $2 \mathrm{~B}$ & $Q S y 2 B$ & Xbarc101-Xcwem55 & 77.4 & 9.2 & -0.98 & 3.88 \\
\hline & $3 \mathrm{~A}$ & $Q \operatorname{Sy} 3 A$ & Xwmc264-Xcfa2193 & 139.9 & 8.42 & 0.72 & 2.1 \\
\hline & 4B & $Q \operatorname{Sy} 4 B$ & Xwmc657-Xwmc48 & 17.7 & 11.27 & -1.55 & 9.76 \\
\hline & $4 \mathrm{D}$ & $Q S y 4 D$ & Xbarc334-Xwmc331 & 3.1 & 11.68 & 1.24 & 6.22 \\
\hline & $5 \mathrm{~A} 2$ & QSy $5 A 2$ & Xcfe026.1-Xcwem 32.2 & 0 & 8.99 & 0.2 & 2.89 \\
\hline
\end{tabular}

${ }^{a}$ Additive effect, a positive value indicates that the allele from HP3 increases the trait value; a negative value indicates that the allele from YM57 increases the trait value. ${ }^{b}$ Proportion of the phenotypic variation explained by the QTL.

Four QTL controlling BY were located on chromosomes 3A, 4B, 4D, and 5A2 in the two environments, accounting for 2.57 to $10.87 \%$ of the phenotypic variation. Both parents carried QTL alleles that increased phenotypic values. A major QTL, QBy4D, with its allele originating from HP3, was mapped to the Xbarc334-Xwmc331 interval on chromosome 4D and explained $10.87 \%$ of the phenotypic variation. The QTL $Q B y 3 A$, originating from HP3, had positive effects on BY. However, the YM57 alleles of $Q B y 4 B$ and $Q B y 5 A 2$ increased BY.

Four QTL with additive effects significantly influencing GY were located on chromosomes 2D, 4B, 4D, and 7B2. All four QTL could account for $22.30 \%$ of the phenotypic variation. The QTL $Q G y 4 D$, with its allele originating from HP3, made the highest contribution explaining $8.74 \%$ of the phenotypic variation. Two QTL ( $Q G y 4 B$ and $Q G y 7 B 2)$ had negative effects on GY and were contributed by YM57 alleles, while the locus $Q G y 2 D$ had positive effects on GY and was transmitted by HP3 alleles. This suggested that alleles increasing GY were dispersed within the parents, resulting in small differences in phenotypic values between the parents and transgressive segregants among the $\mathrm{DH}$ lines.

Five regions on chromosomes $2 \mathrm{~B}, 3 \mathrm{~A}, 4 \mathrm{~B}, 4 \mathrm{D}$, and $5 \mathrm{~A} 2$, which were associated with SY, were identified in both Tai' an and Jiyuan. These loci explained 2.10 to $9.76 \%$ of the phenotypic variation. Of these loci, two of the favorable alleles ( $Q S y 2 B$ and $Q S y 4 B)$, deriving 
from YM57, decreased SY and had an additive effect of 0.98 and 1.55, respectively, whereas the others ( $Q S y 3 A, Q S y 4 D$, and $Q S y 5 A 2)$, originating from HP3, increased SY. Furthermore, $Q S y 4 D$, which was detected in the interval Xbarc334-Xwmc331, had the most significant effect, accounting for $9.76 \%$ of the phenotypic variance.
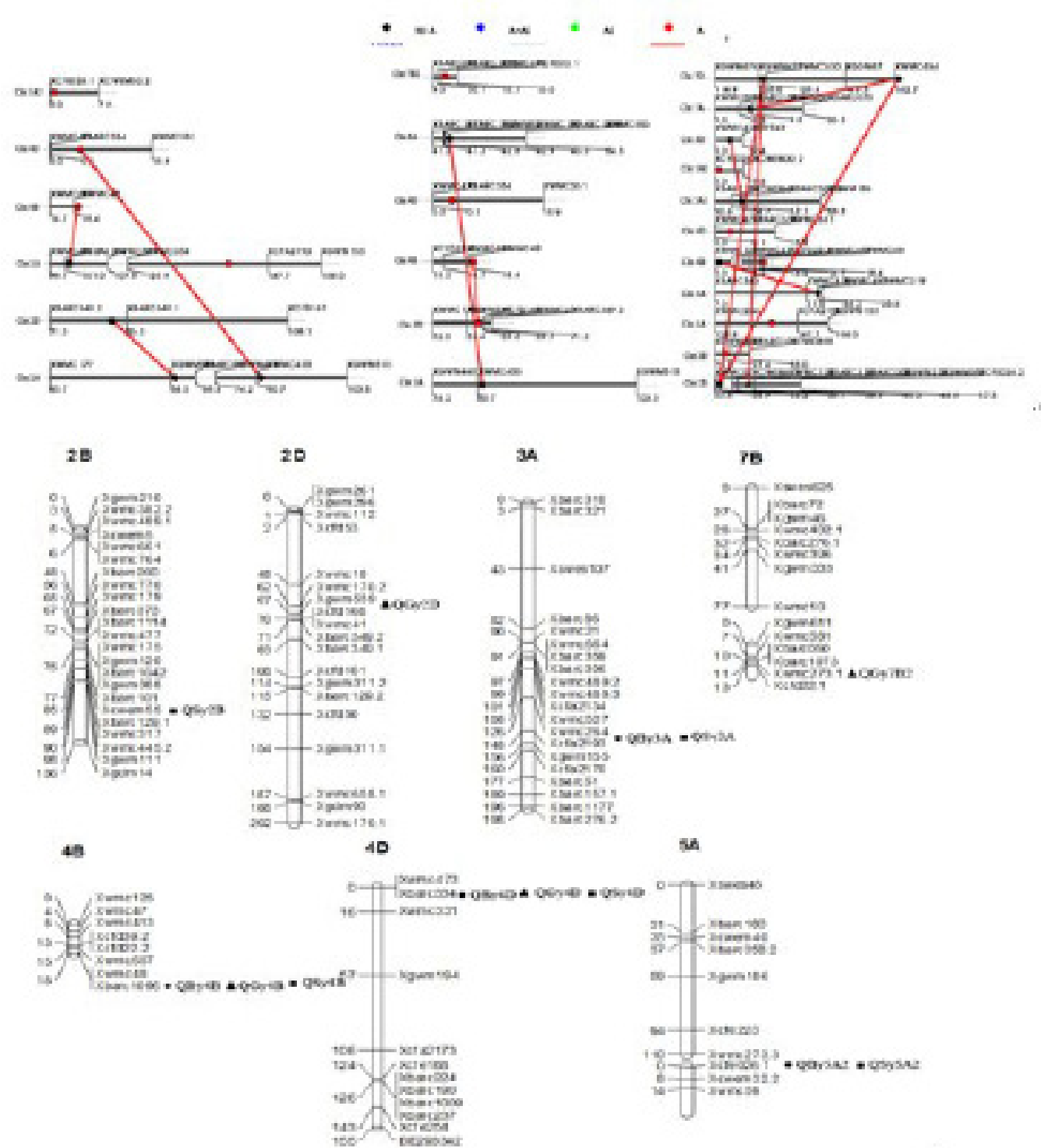

- QTI for BY $\mathbf{Q T L}$ for GY $\approx$ QTI for SY

Figure 2. Positions of additive QTL and epistatic QTL conferring biomass yield (BY), grain yield (GY), and straw yield (SY) at Tai' an and Jiyuan in 2008. QTL listed on the top are epistatic, those below are additive. 


\section{Additive $\mathrm{x}$ additive epistatic interactions}

A total of 11 significant additive-by-additive epistatic interactions were detected for BY, GY, and SY, of which three and eight displayed effects in favor of the parental and recombinant genotype combinations, respectively (Table 4).

Table 4. Additive $\mathrm{x}$ additive epistatic QTL for biomass yield (BY), grain yield (GY), and straw yield (SY) detected at Tai'an and Jiyuan in 2008.

\begin{tabular}{|c|c|c|c|c|c|c|c|c|c|c|}
\hline Traits & romosome & QTL & Marker intervals & Position & Chromosome & QTL & Marker intervals & Position & $\mathrm{AA}^{\mathrm{a}}$ & $\mathrm{R}^{2}(\%)^{\mathrm{b}}$ \\
\hline \multirow[t]{3}{*}{ BY } & $2 \mathrm{~A}$ & $Q B y 2 A-1$ & Xgwm558-Xbarc015 & 64.5 & $2 \mathrm{D}$ & $Q B y 2 D$ & Xbarc349.2-Xbarc349.1 & 80.0 & -1.15 & 2.22 \\
\hline & $2 \mathrm{~A}$ & $Q B y 2 A-2$ & Xwmc455-Xgwm515 & 80.7 & $4 \mathrm{D}$ & $Q B y 4 D$ & Xbarc334-Xwmc331 & 4.1 & 2.25 & 8.46 \\
\hline & $3 \mathrm{~A}$ & $Q B y 3 A-2$ & Xcfa2134-Xwmc527 & 101.0 & $4 \mathrm{~B}$ & $Q B y 4 B$ & Xwmc48-Xbarc1096 & 18.4 & -1.81 & 5.47 \\
\hline \multirow[t]{2}{*}{ GY } & $2 \mathrm{D}$ & $Q G y 2 D$ & Xgwm539-Xcfd168 & 68.4 & $4 \mathrm{~B}$ & $Q G y 4 B$ & Xwmc48-Xbarc1096 & 18.4 & -0.70 & 3.34 \\
\hline & $2 \mathrm{~A}$ & $Q G y 3 A$ & Xwmc455-Xgwm515 & 80.7 & $6 \mathrm{~A}$ & $Q G y 6 A$ & Xbarc1055-Xwmc553 & 43 & 0.88 & 5.28 \\
\hline \multirow[t]{6}{*}{ SY } & $1 \mathrm{~B}$ & $Q S y 1 B-1$ & Xcwem6.1-Xwmc128 & 34.9 & $7 \mathrm{D}$ & $Q S y 7 D-1$ & Xwm437-Хwmc630.1 & 126.6 & 1.54 & 9.53 \\
\hline & $1 \mathrm{~B}$ & $Q S y 1 B-1$ & Xcwem6.1-Xwmc128 & 34.9 & $7 \mathrm{D}$ & $Q S y 7 D-2$ & Xgdm67-Xwmc634 & 162.5 & -0.66 & 1.78 \\
\hline & $1 \mathrm{~B}$ & $Q S y 1 B-2$ & Xgwm218-xgwm582 & 42.2 & $7 \mathrm{D}$ & $Q S y 7 D-1$ & Xwm437-Xwmc630.1 & 126.6 & -0.75 & 2.27 \\
\hline & $4 \mathrm{~A}$ & $\overline{Q S y} 4 A$ & Xwmc313-Xwmc497 & 33.5 & $4 \mathrm{~B}$ & $Q S y 4 B$ & Xwmc125-Xwmc47 & 0.0 & -1.05 & 4.48 \\
\hline & $5 \mathrm{~A} 1$ & QSy $5 A 1$ & Xcwem40-Xbarc358.2 & 36.7 & $6 \mathrm{D}$ & $Q S y 6 D$ & $X w m c 412.1-X c f d 49$ & 3.0 & -1.22 & 4.99 \\
\hline & $7 \mathrm{~A}$ & QSy7A & Xbarc157.2-Xgwm60 & 8.3 & 7D & $Q S y 7 D$ & Xgdm67-Xwmc634 & 162.5 & -0.50 & 1.00 \\
\hline
\end{tabular}

${ }^{a}$ Additive $\mathrm{x}$ additive effect, a positive value indicates that the effect of the parents' effect is larger than the recombinant effect, and a negative value means that the recombinant effect is larger than the parents' effect. 'Proportion of the phenotypic variation explained by additive $\mathrm{x}$ additive QTL.

Three pairs of epistatic effects were detected for BY, which were located on chromosomes 2A/2D, 2A/4D, and 3A/4B, respectively (Table 4, Figure 2). These QTL explained 2.22 to $8.46 \%$ of the phenotypic variation. The QTL pair $Q B y 2 A-2 / Q B y 4 D$ acted in favor of the parent and had the largest effect, contributing a BY of 2.25 and accounting for $8.46 \%$ of the phenotypic variance. The other two QTL pairs in favor of the recombinant type, $Q B y 2 A-1 /$ $Q B y 2 D$ and $Q B y 3 A-2 / Q B y 4 B$, had an effect of 1.15 and 1.81, and together explained $7.69 \%$ of the phenotypic variation.

Two pairs of epistatic interactions were common in both environments for GY. One interaction $(Q G y 3 A / Q G y 6 A)$ acted to increase the values of the parental types, and the other $(Q G y 2 D /$ $Q G y 4 B$ ) acted in the opposite direction, that is, recombinant effects were larger than parental effects. These QTL accounted for 5.28 and $3.34 \%$ of the phenotypic variance, respectively.

Six pairs of epistatic interactions on chromosomes 1B/7D, 4A/4B, 5A1/6D, and $7 \mathrm{~A} / 7 \mathrm{D}$, were identified for $\mathrm{SY}$. The three additive $\mathrm{x}$ additive epistatic interactions of $1 B / 7 D$ acted in favor of the parental and recombinant types, respectively. The three other epistatic interactions acted by increasing the values of the recombinant types, which explained 1.00 to $4.99 \%$ of the phenotypic variation.

\section{DISCUSSION}

\section{Relationship among BY, GY, and SY}

It is expected that crop yield will continue to increase as there are no indications as yet that the yield of cereal crops are approaching limiting conditions (Evans and Fisher, 1999). However, the days to maturity of existing cultivars already match the short growing season 
and the harvest index is already at a high level so that enhancing it further would be detrimental to yield (Takeda and Frey, 1987). Therefore, higher rates of biomass accumulation will be the main option to obtain higher yields (Zhang et al., 2004).

In the present study, BY showed a significantly positive phenotypic correlation with both GY and SY. GY also showed a positive correlation with SY. At the same time, HP3 had a better stability harvest index of 0.44 and 0.45 in Tai' an and Jiyuan, respectively, while those of YM57 were 0.40 and 0.42 . This finding indicates that a genotype with increased BY could be obtained by increasing GY and SY of the positive alleles for parents via markerassisted selection. However, Liu et al. (2006) found that BY was positively correlated with SY but was uncorrelated with GY. Similarly, SY was found to be negatively correlated with GY in rice. Annicchiarico et al. (2005) reported that GY was positively correlated with aerial biomass $(\mathrm{r}=0.61, \mathrm{P}<0.01)$, but was not correlated with $\mathrm{SY}$ in semi-dwarf durum wheat cultivars. Different crops and different wheat cultivar features may explain the different results of correlation coefficients for BY, SY, and GY. Therefore, in order to increase BY, the wheat cultivar should show positive correlations among BY, GY, and SY, as well as a high harvest index. Using these traits, it will now be possible to improve super-high yield wheat breeding programs.

\section{QTL of BY, GY, and SY}

QTL for BY, GY, and SY were detected on chromosomes 2B, 2D, 3A, 4B, 4D, 5A2, and 7B2 in two environments in the present study. Many QTL affecting yield have been reported on all chromosomes, with the exceptions of chromosomes $3 \mathrm{D}$ and $5 \mathrm{D}$, in previous studies, and no significant gene by environment interactions were examined (Huang et al., 2006; Cuthbert et al., 2008; McIntyre et al., 2010). The most significant QTL simultaneously identified for BY, GY, and SY in the current study were located on chromosomes 4B and 4D (Figure 2). In fact, chromosomes 4B and 4D are known to carry a number of major genes affecting plant height, yield productivity, and yield components (Huang et al., 2006). Therefore, the QTL on 4B and 4D should be considered to increase wheat biomass, grain, and straw in wheat molecular breeding.

Marza et al. (2006) and Quarrie et al. (2005) discovered that a number of major genes affecting yield productivity were located on chromosome $5 \mathrm{AL}$ in a similar position to chromosome 5A.2. In the present study, QTL ( QBy5A2 and QSy5A2) on 5A2 were detected for BY and SY in the same regions for Xcfe026.1-Xcwem32.2 (Figure 2). Quarrie et al. (2005) and Cuthbert et al. (2008) showed two-grain yield QTL clusters on chromosomes 7A and 7B around the Xwmc273 locus. We detected a QTL $(Q B y 7 B)$ close to Xwmc273.1 on 7B2 with significant effects on GY (Figure 2), which had a similar interval to that reported by Quarrie et al. (2005). Unfortunately, however, we failed to detect any QTL on chromosome 7A. On chromosome 2D, a QTL that increased GY was detected using $402 \mathrm{DH}$ lines from the spring wheat cross Superb (high yielding)/BW278 (low yielding) by Cuthbert et al. (2008). The QTL on 2D affecting GY was also identified in our study (Figure 2). Zhang et al. (2009) also detected a QTL with significant effects on GY on chromosome 2D. At the same time, we found some new QTL that affected both BY and SY. For example, a QTL detected on chromosome 3A had a great influence on increasing BY and SY in different environments, and explained 3.50 and $2.10 \%$ of the phenotypic variation, respectively. 


\section{Relationship between additive effects and additive $\mathrm{x}$ additive epistatic effects of QTL}

Additive effects and additive $\mathrm{x}$ additive epistatic effects are an important factor affecting the phenotypic expression of complex trait genes and genetic variations in populations (Liao et al., 2001). Generally, the additive $\mathrm{x}$ additive interactions detected included all three types of epistatic effects, classified on the basis of whether the QTL involved exhibited their own main effects or not. These types were termed to be epistatic between two additive loci, between an epistatic locus and an additive locus, or between epistatic loci only, which are equivalent to the terms of interactions between QTL, interactions between QTL and background loci, and interactions between complementary loci ( $\mathrm{Li}, 1998)$.

In the present study, a total of 13 QTL with significant additive effects and 11 significant additive $\mathrm{x}$ additive interactions were detected for BY, GY, and SY using a DH population. Among them, seven occurred between QTL showing epistatic effects only. For example, one interaction that occurred between $Q B y 2 A$ and $Q B y 2 D$ was detected for BY, although $Q B y 2 A$ and $Q B y 2 D$ did not have additive effects. Similarly, $Q G y 3 A / Q G y 6 A$ had additive x additive epistatic effects of GY, but neither had additive effects. Two interactions occurred between QTL showing epistatic effects and additive effects. The QTL detected on 4A and 4D indicated epistatic interactions for SY; only $Q S y 4 A$ had additive effects. Two interactions occurred between QTL with additive effects. The additive QTL $Q G y 2 D$ and $Q G y 2 D$ had an epistatic interaction acting on BY. Overall, the detection of additive and additive $\mathrm{x}$ additive effects of a QTL interfered with each other, indicating that the detection of QTL might vary greatly depending on their interactions with other loci in complex traits (Zhuang et al., 2002).

\section{Effect of Rht1 and $R h t 2$ genes}

Dwarfing alleles for reduced plant height and the presence of $R h t$ resulted in a significant effect in biomass yield, most yield components, and agronomic traits (Cuthbert et al., 2008). The effects of Rht dwarfing genes on increasing wheat yield and improving agronomic characteristics have been studied previously (Li et al., 2006). In our study, the significant QTL identified for BY, GY, and SY were located on chromosomes 4B and 4D, which were detected at the similar intervals of Xwmc48-Xbarc1096 and Xbarc334-Xwmc331 as reported in Zhang et al. (2008) using the same DH population, and also had a similar position of Rht1 and Rht2 (Huang et al., 2003). The height reducing genes were significantly associated with BY components under different environments. Rht 1 reduced plant height (Zhang et al., 2008), BY, GY, and SY, accounting for $14.51,6.12,4.01$, and $9.76 \%$ of the phenotypic variance, respectively. Similarly, Rht2 reduced plant height, BY, GY, and SY, accounting for 20.22, 10.87, 8.74, and $6.22 \%$ of the phenotypic variance, respectively (Zhang et al., 2008). These QTL alleles came from the same parents and had the same effects in different environments. In fact, modifications of plant architecture have the potential to enhance biomass production, and plant height is an important component of architecture that is highly correlated with biomass yield (Lübberstedt et al., 1997). New breeding goals and plant ideotypes need to be established for biofuel production systems. The QTL and candidate genes presented here could speed up breeding progress or allow for a more controlled improvement of the trait. Increasing plant height is the most obvious and direct way to impact BY, GY, and SY, although not the only way. In rice, modifications of plant height have shown encouraging results: grain yield increases of $15-44 \%$ 
were demonstrated, providing a favorable scenario for biomass production from crop residues (Wu et al., 2008). Therefore, new genotypes in wheat that increase plant height have the potential to further increase biomass yield and component traits if lodging issues can be mitigated.

\section{QTL for wheat breeding}

The goal of crop genomics is to understand the genetic and molecular basis of all biological processes in plants that are relevant to the species (Vassilev et al., 2006). QTL mapping thus represents the foundation for the selection of markers for crop breeding. First, selection should be applied particularly for QTL with main genetic effects, which will likely show stable performance across different environments. On the one hand, the actions of QTL involved in epistatic interactions always depend on other loci. On the other hand, these QTL could produce varieties adapted to specific ecosystems. Thus, it should be more efficient to select superior genetic combinations rather than to select single genes. Second, selection programs should consider QTL with interaction effects in different environments (QE), which will also provide confidence for breeders prior to undertaking marker-assisted selection for complex traits in the design and implementation of breeding strategies. However, QE interaction effects might not be well applied across various environments (QE interaction effects were not evident in the present study). Third, selection programs should be based on closely linked or pleiotropic QTL. Breeders could possibly simultaneously improve several related traits by selecting such trait-correlated QTL (Liu et al., 2006). In the current study, the closely linked BY or pleiotropic QTL were coincident with yield components, and an increase in plant height suggested that selecting for a yield component could efficiently increase BY. These results will make marker-assisted selection more efficient for improvement of wheat BY traits. Thus, detailed information on loci involved in related traits and their genetic relationships will certainly be helpful to improve biomass potential and future biofuel production by deliberately manipulating these loci through marker-assisted selection.

\section{ACKNOWLEDGMENTS}

Research supported by the State Key Development Program of Basic Research of China (“973”, \#2009CB118301) and the National Natural Science Foundation of China (\#30971764). The authors thank the Institute of Jiyuan Agricultural Science, Henan, China, for helping to plant the research materials and to collect the phenotypic data in Jiyuan, and Professor Walker C.E. (Kansas State University of America) for his kind guidance on this manuscript.

\section{REFERENCES}

Anhalt UCM, Heslop-Harrison JS, Piepho HP, Byrne S, et al. (2009). Quantitative trait loci mapping for biomass yield traits in a Lolium inbred line derived F2 population. Euphytica 170: 99-107.

Annicchiarico P, Abdeliaoui Z, Kelkouli M and Zerargui H (2005). Grain yield, straw yield and economic value of tall and semi-dwarf durum wheat cultivars in Algeria. J. Agric. Sci. 143: 57-64.

Ayala L, Henry M, van Ginkel M, Singh R, et al. (2002). Identification of QTLs for BYDV tolerance in bread wheat. Euphytica 128: 249-259.

Borlaug NE and Dowswell CR (1997). The Acid Lands: One of Agriculture's Last Frontiers. In: Plant-Soil Interactions at Low pH (Moniz AC, Furlani AMC, Schaffert RE, Fageria NK, et al., eds.). Brazilian Soil Science Society Brazil, Campinas, 5-15. 
Cuthbert JL, Somers DJ, Brule-Babel AL, Brown PD, et al. (2008). Molecular mapping of quantitative trait loci for yield and yield components in spring wheat (Triticum aestivum L.). Theor. Appl. Genet. 117: 595-608.

Doerge RW (2002). Mapping and analysis of quantitative trait loci in experimental populations. Nat. Rev. Genet. 3: 43-52. Evans LT and Fisher RA (1999). Yield potential: its definition, measurement, and significance. Crop Sci. 39: 1544-1551.

Gabrielle B and Gagnaire N (2008). Life-cycle assessment of straw use in bio-ethanol production: A case study based on biophysical modelling. Biomass Bioenergy 32: 431-441.

Guo CQ, Bai ZA, Liao PA and Jin WK (2004). New high quality and yield wheat variety Yumai 57. Chin. Seed Industry 4: 54.

Hai Y and Kang MH (2007). Breeding of a new wheat variety Huapei 3 with high yield and early maturing. J. Henan Agric. Sci. 5: 36-37.

Huang X, Qian Q, Liu Z, Sun H, et al. (2009). Natural variation at the DEP1 locus enhances grain yield in rice. Nat. Genet. 41: 494-497.

Huang XQ, Coster H, Ganal MW and Roder MS (2003). Advanced backcross QTL analysis for the identification of quantitative trait loci alleles from wild relatives of wheat (Triticum aestivum L.). Theor. Appl. Genet. 106: 1379-1389.

Huang XQ, Cloutier S, Lycar L, Radovanovic N, et al. (2006). Molecular detection of QTLs for agronomic and quality traits in a doubled haploid population derived from two Canadian wheats (Triticum aestivum L.). Theor. Appl. Genet. 113: 753-766.

Kirigwi FM, van Ginkel M, Brown-Guedira G, Gill BS, et al. (2007). Markers associated with a QTL for grain yield in wheat under drought. Mol. Breed. 20: 401-413.

Kuchel H, Williams KJ, Langridge P, Eagles HA, et al. (2007). Genetic dissection of grain yield in bread wheat. I. QTL analysis. Theor. Appl. Genet. 115: 1029-1041.

Lal R (2005). World crop residues production and implications of its use as a biofuel. Environ. Int. 31: 575-584.

Li JZ, Huang XQ, Heinrichs F, Ganal MW, et al. (2006). Analysis of QTLs for yield components, agronomic traits, and disease resistance in an advanced backcross population of spring barley. Genome 49: 454-466.

Li M, Guo XH, Zhang M, Wang XP, et al. (2010). Mapping QTLs for grain yield and yield components under high and low phosphorus treatments in maize (Zea mays L.). Plant Sci. 178: 454-462.

Li ZK (1998). Molecular Analysis of Epistasis Affecting Complex Traits. In: Molecular Dissection of Complex Traits (Paterson AH, ed.). CRC Press, Boca Raton, 119-130.

Li ZK, Luo LJ, Mei HW, Wang DL, et al. (2001). Overdominant epistatic loci are the primary genetic basis of inbreeding depression and heterosis in rice. I. Biomass and grain yield. Genetics 158: 1737-1753.

Liao CY, Wu P, Hu B and Yi KK (2001). Effects of genetic background and environment on QTLs and epistasis for rice (Oryza sativa L.) panicle number. Theor. Appl. Genet. 103: 104-111.

Liu GF, Yang J and Zhu J (2006). Mapping QTL for biomass yield and its components in rice (Oryza sativa L.). Yi Chuan Хие Bao 33: 607-616.

Lübberstedt T, Melchinger AE, Schon CC, Uhz H, et al. (1997). QTL mapping in testcrosses of European flint lines of maize. I. Comparison of different testers for forage yield traits. Crop Sci. 37: 921-931.

Maccaferri M, Sanguineti MC, Corneti S, Ortega JL, et al. (2008). Quantitative trait loci for grain yield and adaptation of durum wheat (Triticum durum Desf.) across a wide range of water availability. Genetics 178: 489-511.

Marza F, Bai GH, Carver BF and Zhou WC (2006). Quantitative trait loci for yield and related traits in the wheat population Ning 7840 x Clark. Theor. Appl. Genet. 112: 688-698.

McIntyre CL, Mathews KL, Rattey A, Chapman SC, et al. (2010). Molecular detection of genomic regions associated with grain yield and yield-related components in an elite bread wheat cross evaluated under irrigated and rainfed conditions. Theor. Appl. Genet. 120: 527-541.

Pfeiffer WH, Sayre KD and Reynolds MP (2000). Enhancing Genetic Gain Yield Potential and Yield Stability in Durum Wheat. In: Durum Wheat Improvement in the Mediterranean Region: New Challenges (Royo C, Nachit MM, Di Fonzo N and Araus JL, eds.). IAMZ. Options Mediterraneennes, Zaragoza, 83-93.

Quarrie SA, Steed A, Calestani C, Semikhodskii A, et al. (2005). A high-density genetic map of hexaploid wheat (Triticum aestivum L.) from the cross Chinese Spring $\mathrm{X}$ SQ1 and its use to compare QTLs for grain yield across a range of environments. Theor. Appl. Genet. 110: 865-880.

Somers DJ, Isaac P and Edwards K (2004). A high-density microsatellite consensus map for bread wheat (Triticum aestivum L.). Theor. Appl. Genet. 109: 1105-1114.

Takeda K and Frey KJ (1987). Improving grain yield in backcross populations from Arena sativa $\mathrm{x}$ A. sterilis matings by using independent culling for harvest index and vegetative growth index or unit straw weight.. Theor. Appl. Genet. 74: 659-665.

Vassilev D, Nenov A, Atanassov A, Dimov G, et al. (2006). Application of bioinformatics in fruit plant breeding. J. Fruit Ornam. Plant Res. 14 (Suppl 1): 145-162. 
Wang DL, Zhu J, Li ZKL and Paterson AH (1999). Mapping QTLs with epistatic effects and QTL x environment interactions by mixed linear model approaches. Theor. Appl. Genet. 99: 1255-1264.

Wu CY, Trieu A, Radhakrishnan P, Kwok SF, et al. (2008). Brassinosteroids regulate grain filling in rice. Plant Cell 20: 2130-2145.

Xue DW, Zhou MX, Zhang XQ, Chen S, et al. (2010). Identification of QTLs for yield and yield components of barley under different growth conditions. J. Zhejiang Univ. Sci. B 11: 169-176.

Yang J and Zhu J (2005). Methods for predicting superior genotypes under multiple environments based on QTL effects. Theor. Appl. Genet. 110: 1268-1274.

Zhang K, Tian J, Zhao L and Wang S (2008). Mapping QTLs with epistatic effects and QTL x environment interactions for plant height using a doubled haploid population in cultivated wheat. J. Genet. Genomics 35: 119-127.

Zhang K, Zhang Y, Chen G and Tian J (2009). Genetic analysis of grain yield and leaf chlorophyll content in common wheat. Cereal Res. Communications 37: 499-511.

Zhang ZH, Li P, Wan LX, Hu ZL, et al. (2004). Genetic dissection of the relationships of biomass production and partitioning with yield and yield related traits in rice. Plant Sci. 167: 1-8.

Zhuang JY, Fan YY, Rao ZM, Wu JL, et al. (2002). Analysis on additive effects and additive-by-additive epistatic effects of QTLs for yield traits in a recombinant inbred line population of rice. Theor. Appl. Genet. 105: 1137-1145. 